\title{
A Robust Road Sign Recognition Using Segmentation with Morphology and Relative Color
}

\author{
（モフォロジー演算と相対色によるセグメンテイションを用いたロバストな交通標識の認識）
}

Thi Thi Zin ${ }^{\dagger}$, and Hiromitsu Hama (member) ${ }^{\dagger}$

\begin{abstract}
We propose a robust road sign recognition system under various illumination conditions. The proposed approach has two steps: segmentation and recognition. The segmentation, which is the focus of this paper, is performed using morphological operations and relative color. The segmented regions are recognized by a template matching method using modified standard deviation. The algorithm works for various types of circular and pentagonal road signs. In experiments under various illumination conditions, the segmentation rate was $100 \%$ in the daytime and evening and $80 \%$ even in the nighttime, and the recognition rate was $100 \%$ for all of the segmented regions under all illumination conditions. The effectiveness of the proposed system was confirmed through experiments using 200 images of road signs taken under a great variety of illumination conditions including fog and light rain.
\end{abstract}

Key words: Segmentation, Robust recognition, Road signs, Morphology, Relative color, Template matching

\section{Introduction}

The road signs and traffic signals provide visual information for safe and smooth driving to drivers. But, the driver's carelessness is often the primary factor of traffic accidents. Sometimes, due to weather conditions or viewing angles, they fail easily to be noticed until it is too late. The robust road sign recognition ${ }^{1) \sim 10)}$ is necessary for driver support systems, which should work as a driver's copilot. Road signs are designed so that they can stand out in a typical road scenario and be easily detected by the combination of distinctive colors and shapes ${ }^{1112)}$. But they are often deteriorated by weather conditions and frequently occluded partially by other vehicles, trees and so on. So, it is relatively easy to recognize them under good conditions, but very difficult under bad conditions. It means that color information is sensitive to the change of weather conditions, lighting conditions and so on. Therefore, shape information has been often used together to solve this kind of problems ${ }^{13)}$. Some ${ }^{14) 15)}$ used a neural network for classification, and others ${ }^{16) 17)}$ used a template matching method. Hsu and Huang ${ }^{16)}$ implemented an automatic road sign recognition system using Matching Pursuit (MP) filters.

\footnotetext{
Received September 17, 2004; Final received May 2, 2005; Accepted June 15, 2005

† Graduate School of Engineering, Osaka City University

(Sugimoto 3-3-138, Sumiyoshiku, Osaka 558-8585, Japan, TEL 066605-2772)
}

Paclik and Novovicova ${ }^{17)}$ used cross-correlation methods for road sign classification. Gavrila ${ }^{18)}$ proposed a multi-feature hierarchical algorithm to match $N$ templates with an image using distance transform. They used the coarse-to-fine search for it, and grouped the $N$ templates into template hierarchies based on similarity.

Almost all of road sign recognition (RSR) systems have been developed under good illumination conditions. In this paper, special attention is paid to robustness and flexibility under bad conditions. Our objectives are such scenes as shown in Fig.1. The proposed algorithm can detect all of these road signs, even if they are under bad illumination conditions, slightly titled, deteriorated or partially occluded. Two methodological approaches are adopted: (1) morphology and morphological gradient and (2) relative color. Shape and color information are used complementarily to improve the performance.

Our proposed robust RSR system is composed of two stages: segmentation and recognition. At the segmentation stage, the success rate was $100 \%$ in the daytime and evening including fog and light rain, and $80 \%$ even though in the night time. Difficulties for segmentation are caused by color deterioration with age, and reflections from close street lights and so on. An example of failure case for segmentation is shown in Fig.2. Since recognition is easier than segmentation, the recognition rate of the segmented images was $100 \%$ in the daytime, 
evening and nighttime. Although the segmentation of Fig.2(a) ended in failure, the recognition of the manually segmented road signs in Fig.2(b) was successful. Even though in such blurred images, the proposed algorithm can give the perfect recognition rate. According to the experimental results, the use of modified standard deviation in template matching is very effective and powerful compared with conventional recognition methods.

The overall flow chart for a RSR system is shown in Fig.3. In Section 2, the segmentation method using morphology and relative color is presented. The recognition method using standard deviation is described in Section 3. In Section 4, the effectiveness of our proposed method is confirmed through experimental results under various illumination conditions and occluded conditions. Finally, conclusions and future works are discussed in Section 5.

\section{Segmentation by morphology and rel- ative color}

\subsection{Structuring elements and morphological gradients}

Morphological gradients are often used for edge detection, segmentation, sharpening and so on. In this paper, they are used for preprocessing of segmentation. Some morphological segmentation methods, such as watershed segmentation, are utilized for edge detection and give the good result for segmentation of unknown shape objects. But in this paper, the objects are road signs which have known shapes such as circles and pentagonshapes. According to the types of road signs, we choose the optimal shapes of structuring elements (SE) as follows:

- disks and arcs for circular signs with a thick red ring,

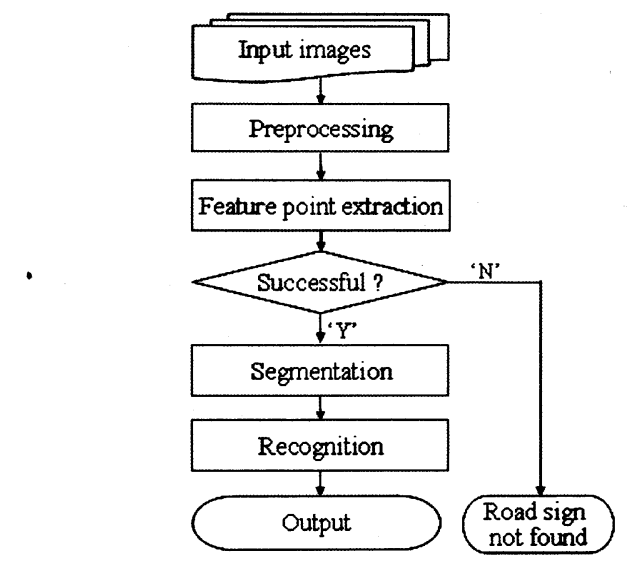

Fig.3 Overall flow chart for a RSR system.
- lines for circular signs without a thick red ring,

- triangle and combination of lines for pentagonal signs.

Table 1 shows the notations of SEs and their meaning which will be used in the next part of this paper. Using appropriate SEs, a morphological gradient is performed directly over color input images. The obtained gradient in RGB is changed into intensity using Eq.(1).

$$
I=0.3 R+0.59 G+0.11 B .
$$

In addition to traditional basic morphological gradients (BMG), combinatorial morphological gradients $(\mathrm{CMG})$ are introduced here.

\section{(1) Basic morphological gradient}

The BMG $\rho_{B}$ by a SE $B$ is defined as follow:

$$
\rho_{B}=\delta_{B}-\varepsilon_{B},
$$

where $\delta_{B}$ and $\varepsilon_{B}$ are dilation and erosion, respectively. SEs are binary and flat, and the black and white regions of an input image $f$ in Fig. 4 and Fig.5 mean relatively large and small values, respectively. The origin of a $\mathrm{SE}$ is expressed by a red point. Let $R_{1}$ and $R_{2}$ be the radii of interior and exterior circles, respectively, and suppose $R_{1}>R_{2}-R_{1}$. This supposition is right as long as concerning circular road signs in Japan. The results of BMGs using various sizes of $r$ enlarging gradually are classified into 5 cases, and some of them are shown in Fig.4. The difference of $R_{2}-R_{1}$ can be got from Case 2 , and the center from Case 4.

Case 1: $\quad r<\frac{1}{2}\left(R_{2}-R_{1}\right) \quad \rightarrow$ double rings, Case 2: $\quad r=\frac{1}{2}\left(R_{2}-R_{1}\right) \quad \rightarrow$ double rings and middle white circle,

Case 3: $\quad \frac{1}{2} R_{2}>r>\frac{1}{2}\left(R_{2}-R_{1}\right) \rightarrow$ single ring,

Case 4: $\quad r=R_{1} \quad \rightarrow$ white point,

Case 5: $\quad r>R_{1} \quad \rightarrow$ painted out.

Here, we define the arc shape SE as shown in Fig.5 for the inner side top point detection of circular sign's upper regions. The arc shape is approximated by piecewise straight lines. In order to get stable results of relative colors, the inner side point is adopted instead of the outer one. When rotating the SE by 90 degrees three times, we can also get SEs to detect the inner side points of left, bottom and right regions of the ring. These SEs are used to detect road signs with thick red rings.

\section{(2) Combinatorial morphological gradient}

Two types of CMGs using line shape SEs are introduced. They are composed of Boolean operations, 'OR' and 'AND'. An example of the first type is expressed in 
Fig.6. It means that the BMG $\rho_{1}$ of the upper line is large because of a border line region. Since the middle line is in a uniform color region, the BMG $\rho_{2}$ becomes small. And the BMG $\rho_{3}$ of the lower one becomes large due to including different colors. To detect the inner side top point of this road sign without a thick red ring, each CMG is combined as follows:

( $\rho_{1}$ is large) $\operatorname{AND}\left(\rho_{2}\right.$ is small) $\operatorname{AND}\left(\rho_{3}\right.$ is large).

Next, the second type of CMG is introduced to detect the bottom corners of pentagonal road signs. According

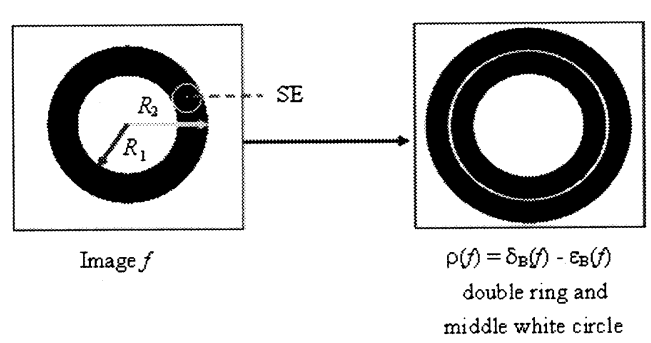

(a)

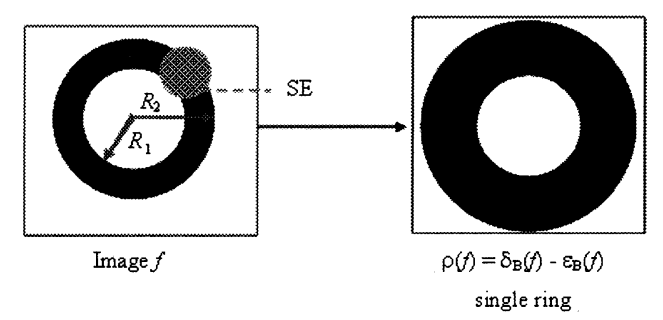

(b)

Fig.4 BMGs with disk shape SEs: (a) Case 2, (b)Case 3.

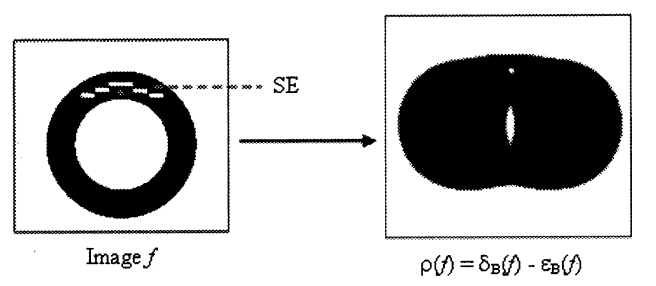

Fig.5 BMGs with an arc shape SE.

Table 1 Various SEs.

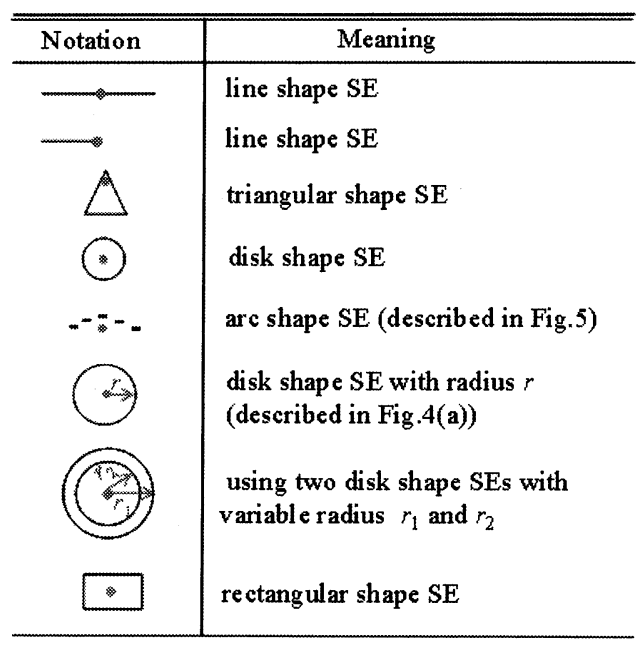

For each $\mathrm{SE}$, the red color pix el (*) is the origin. to the traditional morphology, the erosion and dilation are defined by the minimum and maximum values of the pixels within the region $\mathrm{SE}$, respectively. In this paper, the $i^{\text {th }}$ minimum $\left(E_{i}\right)$ and the $i^{\text {th }}$ maximum $\left(D_{i}\right)$ are adopted, so called 'rank filter'. Then the gradient $G_{i}$ is defined as:

$$
G_{i}=D_{i}-E_{i}
$$

where, $G_{i} \geqq 0$ for $\forall i$, and $G_{i} \leqq G_{j}$ if $i>j$. When $i=1$, $G_{i}$ is the same with BMG $\rho$. The newly defined gradient can easily remove spike noise and tolerate some transformations as shown in Fig.7(a). Since $G_{3}$ in Fig.7(a) has the small value, we can segment this region as one region in spite of including big spike noises. Similarly, we can detect the bottom corner region by $G_{i}$ even if it is rotated just a little as shown in Fig.7(b). For example, the modified dilation $D_{i}$ at $\left(x_{0}, y_{0}\right)$ is defined as follows:

$$
\begin{aligned}
D_{i} & =\max \left(X_{0}, Y_{0}\right), \\
X_{0} & =i^{t h} \max \left\{a_{k}\right\} \\
Y_{0} & =i^{t h} \max \left\{b_{k}\right\} \\
a_{k} & = \begin{cases}\left(x_{k}, y_{0}\right), & \text { if } 1<k<5 \\
\min \left[\left(x_{k}, y_{1}\right),\left(x_{k}, y_{0}\right),\left(x_{k}, y_{-1}\right)\right], & \text { if } 5 \leqq k<11\end{cases} \\
b_{k} & = \begin{cases}\left(x_{0}, y_{j}\right), & \text { if } 1<k<5 \\
\min \left[\left(x_{-1}, y_{k}\right),\left(x_{0}, y_{k}\right),\left(x_{1}, y_{k}\right)\right], & \text { if } 5 \leqq k<11\end{cases}
\end{aligned}
$$

where the modified erosion $E_{i}$ is defined similarly with $D_{i}$ exchanging max for min. The proposed algorithm is intended to rotation invariant. Therefore, the allowance of rotation is about 20 degrees. The values of $k$ and $i$ are determined according to pre-experiment results.

Thus, all of the feature points of target road signs are detected without overlooking, though some outliers may be often included. Moreover, the points give the smallest morphological gradient values in and around. There are left some noises with small gradient values especially in the similar regions to the target road signs or the background regions like sky. To solve this kind of problems, we also propose the relative color method which is introduced in the next section.

\subsection{Relative color}

One of the most significant characteristics of all road signs is the combination of distinctive colors. Many color segmentation methods have been implemented in RGB color space ${ }^{15) 19)} \mathrm{HSV}^{13)^{20) 21)}}$ and $\mathrm{YUV}^{12)}$. Many researchers used bounding box methods in RGB color space as Definition 1. According to this definition, the road sign segmentation under good illumination conditions may be well done, but it faces big problems under 
bad illumination conditions as shown in Fig.8 and Table 2 . The B components are larger than other components even though in a red color region in Fig.8(a). Moreover, in Fig.8(b), R, G and B components have very small values. So Definition 1 can't solve these kinds of problems.

Most of papers suggested that the HSV color space might be more flexible to the disturbance caused by lighting problems, but it didn't significantly improve any performance in our experiments. Vitabile et al. ${ }^{20}$ ) used dynamic pixel aggregation techniques in HSV color space using Definition 2. The system performance decreased when the processed images are characterized by predominant sets of pixels whose attributes fall into the HSV achromatic and/or unstable areas. Therefore, the system may not solve to some images at nighttime because the pixel values sometimes fall into the HSV achromatic areas $(\mathrm{V}<0.2)$. Here, every pixel in Fig.8(b) is not included in the chromatic area. From these results, it can be seen that the road sign colors can't be detected only by color bounding boxes under bad illumination conditions.

Definition 1 (in RGB color space)

$R E D$ :if $(R>80)$ AND $(R-G>20)$ AND $(R-B>20)$, $W H I T E:$ if $(R>150)$ AND $(G>150)$ AND $(B>150)$.

Definition 2 (in HSV color space)

Chromatic:

$$
0.25<S<0.5 \text { AND } 0.2<V \leqq 0.9,
$$

Unstable chromatic:

$$
S \geqq 0.5 \text { AND } 0.2<V \leqq 0.9,
$$

\section{Achromatic:}

$$
S \leqq 0.25 \text { OR } V \leqq 0.2 \text { OR } V \geqq 0.9 .
$$

In fact, we have already tried the road sign color separation by a linear learning method ${ }^{22) 23}$, and looked into the color nature of road signs. The road sign colors, such as red, blue and white, are successfully separated in the three dimensional RGB color space by a hyper-plane in the day time but can't in the nighttime. Therefore, we find out it impossible to solve simultaneously under all illumination conditions even though many color

Table 2 Color components in RGB and HSV space.

\begin{tabular}{l|c|c|c|c|c|c|c}
\hline \hline pixels & $R$ & $G$ & $B$ & $H$ & $S$ & $V$ & Colors \\
\hline (A1) & 100 & 41 & 105 & 0.8203 & 0.6095 & 0.4118 & Red \\
\hline (A2) & 63 & 88 & 188 & 0.6333 & 0.6649 & 0.7373 & Blue \\
\hline (B1) & 105 & 52 & 106 & 0.8302 & 0.5094 & 0.4157 & Red \\
\hline (B2) & 134 & 155 & 202 & 0.6152 & 0.3366 & 0.7922 & While \\
\hline (C1) & 9 & 5 & 6 & 0.0714 & 0.7778 & 0.0353 & Red \\
\hline (C2) & 19 & 19 & 27 & 0.5909 & 0.4231 & 0.1020 & While \\
\hline (D1) & 12 & 6 & 8 & 0.9444 & 0.6000 & 0.0392 & REd \\
\hline (D2) & 17 & 22 & 28 & 0.6154 & 0.4231 & 0.1020 & While \\
\hline
\end{tabular}

bounding boxes are prepared. To solve these problems of Definition 1 and 2 shown in Fig.8(c), we propose the relative color method using Definition 3 and 4 . The circular road signs are classified into two groups, with an inner white region and blue one, as shown in Fig.8(a). The method could give the perfect result to separate road sign color regions in the daytime but a few errors are left in the nighttime.

Definition 3 (absolute red color)

$R E D:$ if $\left(R_{0}>G_{0}+10\right)$ OR $\left(R_{0}>1.5 * G_{0}\right)$ AND $\left(R_{0}>1.2 * B_{0}\right.$ OR $\left(R_{0}>0.9 * B_{0}\right.$ AND $\left.B_{0}>G_{0}+7\right)$,

Definition 4 (relative red colors)

(a) RED pixel and inner WHITE pixel:

if $\left(0.3<\frac{G_{0} * r}{R_{0} * g}<0.65\right.$ AND $\left.0.3<\frac{B_{0} * r}{R_{0} * b}<0.7\right)$.

(b) RED pixel and inner $B L U E$ pixel:

if $\left(\frac{B_{0} * r}{R_{0} * b}<0.4\right.$ AND $\left.0.5<\frac{B_{0} * g}{G_{0} * b}<1.1\right)$.

Here, the threshold values are decided through preexperiments. The color components of the point of interest (POI) are expressed by $\left(R_{0}, G_{0}, B_{0}\right)$ and ones of a neighboring pixel by $(r, g, b)$. By using the color ratio as defined in Definition 4 , it becomes possible to recognize bluish red as red, if it exist at least one dark blued pixel in the neighborhood. Thus, almost all of road sign colors are detected under all illumination conditions even though in the nighttime. Here, we sometimes use the term 'relative color' instead of the combined meaning of Definition 3 and Definition 4. The relative color is very powerful and effective not only for bad illumination conditions, but also for removing noises that are left after morphological operations. Here RGB space is adopted because it gives stable and better results than HSV space through preliminary experiments.

\section{3 Road sign segmentation procedure}

The basic idea for color image segmentation is to detect uniform regions in an original image. The flow chart for segmentation is shown in Fig.9. It includes the following 5 steps:

i) Find the nominated feature region using the above described morphological operations with appropriate SEs over the input color image.

ii) Extract feature points in a region using relative color: 4 points (top, bottom, left, right) for a circular road sign and 3 points (top, left bottom, right bottom) for a pentagonal road sign. If there are more than one feature pixels in one corresponding region, adopt the mean of these pixels. 
Moreover, the adopted feature points are always checked with the opponent feature points. By using their measurement ratio, we can delete noises that are included near the nominated feature region.

iii) Estimate the exterior boundary using the coordinates of 4 points for circles and 3 points for pentagon-shapes. Under occlusion or bad illumination condition, we sometimes get only 3 and 2 feature points, respectively. Although in such cases, the estimation is possible using the ratio of road sign's sub-regions.

iv) Segment the exterior rectangular boundary for all types of road signs.

v) Normalize the size into $64 \times 64$ using linear interpolation.

An example of segmentation procedure is described in Fig.10 using a pentagonal road sign. For pentagonal sign detection, nominated feature region detection is developed for top, left and right corners, respectively. Strictly saying, Fig.10(a) shows a part of the whole input image for simplicity. In Fig.10(b), yellow painted regions represent nominated feature regions for a top corner by morphological operations. In Fig.10(c), the top feature point is expressed in yellow, and the left and right ones in red. Using these three feature points, we can estimate the exterior boundary as shown in Fig.10(d). The first step of segmentation using the exterior boundary is performed, shown in Fig.10(e-i). After this, there are some steps for normalization: normalization of angle (Fig.10(e-ii)), the second segmentation (Fig.10(e-iii)), and normalization of size (Fig.10(f)). The finally segmented image (Fig.10(f)) is used at the

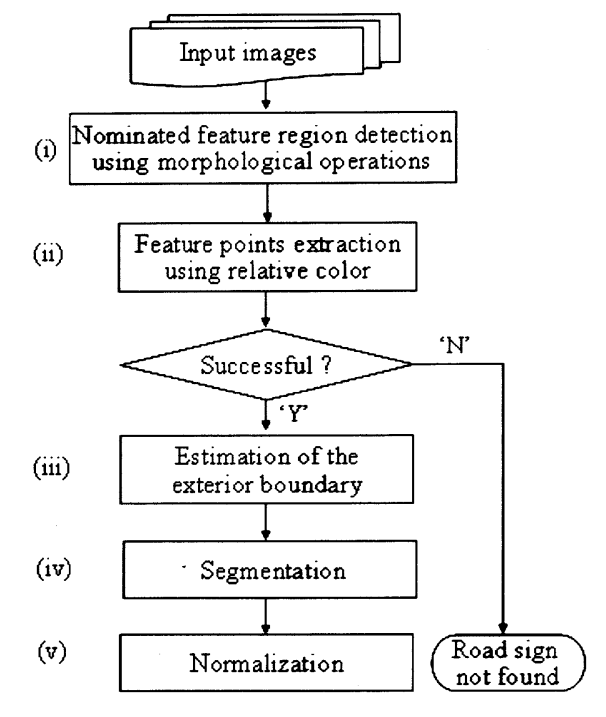

Fig.9 Flow chart of the segmentation procedure. recognition stage. The procedures for segmentation of 3 types of road signs are shown in Table 3 . In this table, Step 1 refers to SEs used in morphological operations and Step 2 to relative colors of POI. The feature points not described in this table can be got in the same way with the same type of points.

\section{(1) Circular road signs with a thick red ring}

In Table 3, the BMGs of the top region of a road sign using SE1 are smaller than other regions. Using the predetermined thresholds, the nominated regions are found and next the BMGs with disk shape SEs (SE2 and SE3) are computed. The positions whose gradients are smaller than the thresholds are painted in pink in Fig.11(a-i), which includes many errors. To remove these errors, the relative color method is applied after this. The colors of POIs are checked at Step 2 using Definition 3. Next, it is checked whether or not there exists at least one pixel in $B$ which satisfies the color ratio of $A$ and $B$ of Definition 4 , or not. The detected top feature points are shown in yellow in Fig.11(a-ii). Similarly, bottom, left and right feature points are also detected as shown in Fig.11(b-i)-(b-iii). Using these points, the road signs can be segmented as shown in Fig.11(c-i) and (c-ii), respectively.

\section{(2) Circular road signs without a thick red ring}

How to use CMGs has already been explained in Fig.6. The next step is the use of relative color. In Table 3 , every pixel of the region $A^{\prime}$ under POI $A$ must have the same color with $A$. By this way, the feature point extractions for top, bottom, left and right are succeed, so we can go to the next steps of Fig.9, segmentation and normalization.

\section{(3) Pentagonal road signs}

An explanatory example of left corner detection is shown in Fig.12: (a) CMG using SE1 in Table 3, (b) the result of the BMGs using SE2 (explained in Fig.7), (c) AND operation using (a) and (b), and (d) after relative color composition ratio on (c). After detecting three feature points (top, left and right corners), we can segment the road sign using the ratio of width and height, and refer to Fig. 10.

\section{Recognition by template matching}

In this section, the road sign recognition method is developed with a new template matching method. All templates are made from the uniform component color region of road signs, and the regions are a little thinner 
than the original regions. Each template with the size of $64 \times 64$ is made from a good and clear road sign. Templates used here are shown in Fig.13(a)-(c). Each road sign is composed of two to four templates according to color components included in it.

For road sign recognition, we have already proposed three methods for template matching ${ }^{24)}$ : (1) standard deviation method, (2) cumulative frequency method, and (3) moving average method which is the best. The recognition rate in the daytime is $100 \%$, in the evening more than $80 \%$ and in the night time nearly $70 \%$, respectively in our past studies. We proposed template matching with CCD (Component Color Distance) for recognition, not considered for segmentation ${ }^{25)}$. However, the above methods were used with the template size $128 \times 128$ and the input signs were manually segmented. In this paper, the input signs are segmented automatically using the proposed segmented method that is already described in Section 2. Moreover, the template size is reduced to $64 \times 64$. The flow chart of recognition procedure is shown in Fig.14. We extend the methods ${ }^{24) 25}$. Let $X_{1}$ and $X_{2}$ be the whole segmented region of a road sign and the matching region of each template, respectively. The black regions of Fig.13(d) and (e) represent $X_{1}$ s for circular and pentagonal road signs, respectively. The black regions of each template of Fig.13(a)-(c) represent $X_{2}$. Whenever the standard deviation $S_{1}$ of $X_{1}$ is large (small), the standard deviation $S_{2}$ of $X_{2}$ is also large (small). In order to get the stable parameters, we adopt the modified standard deviation $S_{2} / S_{1}$ (abbreviated by MSTD) instead of $S_{2}$. Otherwise, it was difficult to get the good matching results for all illumination conditions due to our past experiences. These aspects are shown in Fig. 15. As seen in Fig.15, for STD, $T h_{1}$ and $T h_{2}$ are 5 and 10 , respectively, so multiple matched signs occur for small STD and no matched sign for large STD. If we change $T h_{1}$ to 2 and $T h_{2}$ to 12 , we can solve small STD and large STD case, but multiple matched signs occur again in middle STD as shown in Fig.16. To solve these problems simultaneously, we propose MSTD instead of STD. The effectiveness of MSTD is confirmed by experimental results. The procedures for template matching using MSTD are as follows:

i) Compute MSTDs $S_{R}, S_{G}, S_{B}$ of color components on the black regions of all templates and change into the intensity value by $\mathrm{Eq}(1)$.

ii) Decide 'matched', 'un-decided ' , or 'un-matched' of all templates from the results based on two pre- determined thresholds.

iii) Determine a road sign from the possible pairs of the 'matched' and 'un-decided' templates, and then the 'matched' ones are given priority over the 'un-decided' ones.

According to our experimental results, MSTD can solve for various illumination conditions even though in the nighttime. Based on the sample road signs, two threshold values $T h_{1}$ and $T h_{2}$ were predetermined as 0.3 and 0.5 , respectively. The small MSTD means that the corresponding region has uniform color. Therefore, if MSTD is less than $T h_{1}$, then it is 'matched' and between $T h_{1}$ and $T h_{2}$, then 'undecided', and otherwise, 'un-matched'. Fig.16 shows the results of matching us-
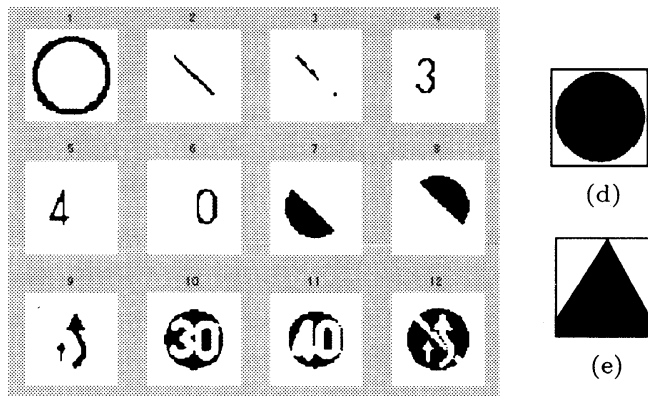

(d)

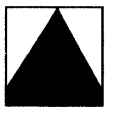

(e)

(a)

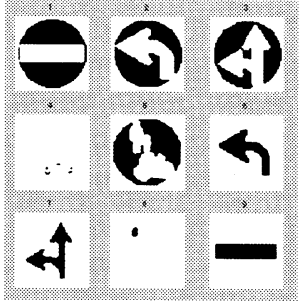

(b)

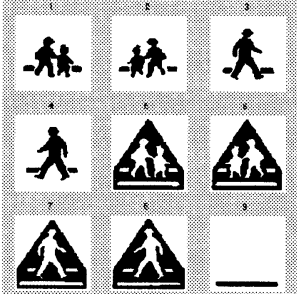

(c)
Fig.13 Templates for:(a) circular road signs with a thick red ring, (b) circular road signs without a thick red ring, (c) pentagonal road signs, (d) $X_{1}$ for (a) and (b), and (e) $X_{1}$ for (c).
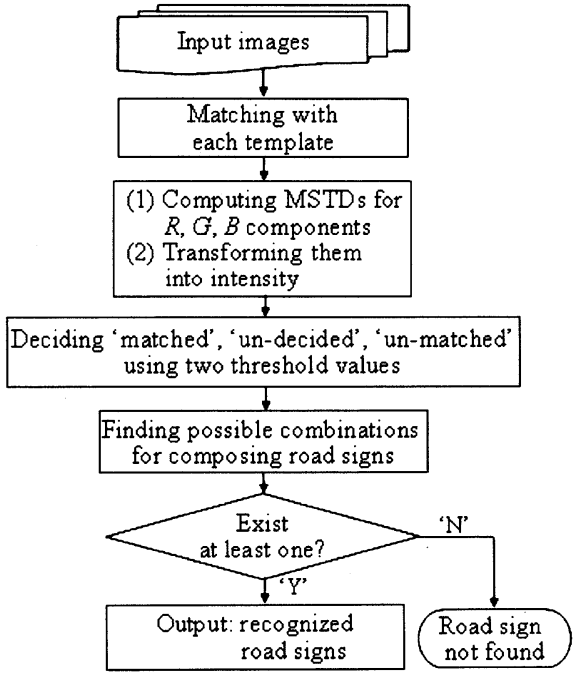

Fig.14 Flow chart of recognition procedure. 
ing MSTD where twelve templates in Fig.13(a) are used. It is seen in Fig.16 that MSTD is stable although STD is easily changeable. In Fig.16(a), there are four 'matched' templates (No.1, 5, 6 and 11), whose MSTDs are less than $T h_{1}$, then they can compose " $40 \mathrm{~km}$ speed limit" sign. Thus, it was impossible to get the perfect recognition rate without the advantage of MSTD.

\section{Experimental Results}

The detail explanations for segmentation and recognition are already described in Section 2 and Section 3, respectively. To evaluate the performance of the proposed RSR system, we used 200 images of road signs taken by digital cameras Nikon D1 and Olympus CAMEDIA C-2500L at the distances of $30 \mathrm{~m}$ to $60 \mathrm{~m}$. It is said that the stopping distance is from $35 \mathrm{~m}$ to $45 \mathrm{~m}$ at a driving speed $60 \mathrm{~km} / \mathrm{hr}$ on an ordinary dry street. Therefore, our system can keep the enough time for safety drive. There are many sizes used in this experiment, the height and width are from 500 to 800 pixels. Among 200 images of road signs, 30 are used for deciding parameters. The algorithm has been programmed in Matlab 6.1, and implemented on a personal computer with AMD Athlon ${ }^{\mathrm{TM}} \mathrm{XP} 2800+$ Processor with IBM/AT compatible and 1GB RAM Memory.

The experiments are conducted on road signs under various illumination conditions. Fig.17 shows the segmented and identified results in the nighttime. On the other hand, there were a few cases that failed in segmentation under very bad illumination conditions because of strong light reflections and extremely blurred images as shown in Fig.2. The results for the proposed RSR system are summarized in Table 4. The segmentation gave the perfect successful rate both in the daytime and in the evening, and $80 \%$ even in the nighttime. The recognition succeeded perfectly over not only automatically segmented signs but also manually segmented signs which ended in failure at the segmentation stage.

\section{Conclusion}

In this paper, a robust RSR system has been developed under various illumination conditions such as day-

Table 4 The experimental results for proposed RSR system.

\begin{tabular}{|c|c|c|c|c|}
\hline \multirow{2}{*}{ condition } & \multirow{2}{*}{$\begin{array}{l}\text { used } \\
\text { signs }\end{array}$} & \multicolumn{2}{|c|}{ segmentation } & \multirow{2}{*}{$\begin{array}{l}\text { Recognition } \\
\text { (success rate) }\end{array}$} \\
\hline & & success rate & failure rate & \\
\hline daytime & 80 & $100 \%$ & - & $100 \%$ \\
\hline evening & 80 & $100 \%$ & - & $100 \%$ \\
\hline \multirow{2}{*}{ nighttime } & \multirow{2}{*}{40} & $80 \%$ & & $100 \%$ \\
\hline & & & $20 \%$ & $100 \%$ \\
\hline
\end{tabular}

time, evening and nighttime. We proposed CMG and relative color for segmentation and a template matching method with MSTD for recognition. Generally speaking, segmentation including detection is much more difficult problem than recognition. So, segmentation was focused in this paper. On the other hand, to design a whole RSR system, both of segmentation and recognition are necessary to be robust. The effectiveness of our proposed system was confirmed by through experiments using 200 images of road signs including blurs and occlusions. It takes about a few seconds for the whole process. The current algorithm is implemented for circular and pentagonal road signs, but it is easily extended to other types of road signs like rectangular and diamond-shaped road signs. Although the size of templates used in this paper is $64 \mathrm{x} 64$, the algorithm was tested and confirmed to work well with a few images using the segmented size $24 \times 24$ under bad conditions. Therefore, it will be extended to smaller size temples in near future. Furthermore, it is the next work to get good results under much worse conditions heavy rain, snow and also under more occluded conditions. For practical use, the algorithm should be implemented in real time by special purpose hardware. Our final goal is to understand overall road environments through video images and to transmit only necessary and sufficient information to drivers for safety and amenity by the way not to disturb the driving operation, for example, voice, vibration and so on, in addition to visual information.

\section{[References]}

1) A. de la Escalera, J.M. Armingol, M. Mata: "Traffic Sign Recognition and Analysis for Intelligent Vehicles", Image \& Vision Computing, 21, 3, pp.247-258 (Mar. 2003)

2) J-Hun Lee, K-Hyun Jo: "Traffic Sign Recognition by Division of Characters and Symbols Regions", in Proc. of the 7th KoreaRussian Int. Symposium, KORUS (2003)

3) J. Miura, T. Kanda, S. Nakatani, Y. Shirai: "An Active Vision System for On-Line Traffic Sign Recognition", IEICE Trans. Info. \& Syst., E85-D, 11, pp.1784-1792 (Nov. 2002)

4) A. de la Escalera, J.M. Armingol, J.M. Pastor, F.J. Rodriguez: "Visual Sign Information Extraction and Identification by Deformable Models for Intelligent Vehicles", IEEE Trans. Intelligent Transportation Systems, 5, 2, pp.57-68 (Jun. 2004)

5) L. Paletta: "Detection of Traffic Signs Using Posterior Classifier Combination", in Proc. of 16th Int. Conference on Pattern Recognition, 2, pp.705-708 (2002)

6) E. Perez, B. Javidi: "Nonlinear Distortion-tolerant Filters for Detection of Road Signs in Background Noise", IEEE Trans. Vehicular Tech., 51, 3, pp.567-576 (May 2002)

7) M.V. Shirvaikar: "Automatic Detection and Interpretation of Road Signs", in Proc of the 36th Southeastern Symposium on Syst. Theory, pp. 413- 416 (Mar. 2004)

8) Y.B. Lauziere, D. Gingras, F.P. Ferrie: "A Model-based Road Sign Identification System", in Proc. of the IEEE Comput. Society Conference on Comput. Vision \& Pattern Recognition, 1, pp.1163-1170 (2001)

9) Jiang Gangyi, Zheng Yi, Choi Tae Young: "Morphological Skeleton Analysis of Traffic Signs on Road", IEEE Int. Conference on Syst., Man \& Cybern., Beijing, China, 1, pp.70-75 (Oct. 1996) 
10) A.L. Yuille, D. Snow, M. Nitzberg: "Signfinder: using color to detect, localize and identify informational signs", 6th Int. IEEE Conference on Comput. Vision (Jan. 1998)

11) L. Priese, V. Rehrmann, R. Schian and R. Lakmann: "Traffic Sign Recognition based on Color Image Recognition", in Proc. IEEE Symp. on Intelligent Vehicles, pp.95-100 (1993)

12) W.G. Shadeed, D.I. Abu-Al-Nadi, M.J. Mismar: "Road traffic Sign Detection in Color Images", in Proc. of the 10th IEEE Int. Conference on Electronic, Circuits \& Syst., 2, pp.890-893 (Dec. 2003)

13) G.Piccioli, E.De Micheli, P. Parodi and M.Campani: "Robust method for road sign detection and recognition", Image \& Vision Computing, 14, 3, pp.209-223 (April 1996)

14) U.Franke, D. Gavrila, S. Gorzig, F. Lindner, F. Paetzold, and C. Wohler: "Autonomous driving goes downtown", IEEE Intelligent Syst. \& Their Applications, 13, 6, pp.40-48 (1998)

15) A. de la Escalera, L. Moreno, M. A. Salichs, J.M. Armingol: "Road Traffic Sign Detection and Classification", IEEE Trans. on Industrial Electronics, 44, 6, pp.848-859 (1997)

16) S.-H.Hsu, C.-L.Huang: "Road Sign Detection and Recognition using Matching Pursuit Method", Image \& Vision Computing, 19, 3, pp.119-129 (Feb. 2001)

17) Paclik P., Novovicova J.: "Road Sign Classification without Color information", in Proc. of 6th conference of Advanced School of Imaging \& Computing ASCI, Lommel, Belgium (2000)

18) D.M.Gavrila: "Multi-feature Hierarchical Template Matching Using Distance Transforms", in Proc. IEEE Int. Conference on Pattern Recognition, Brisbane, Auatralia (1998)

19) Lukas Sekanina, Jim Torresen: "Detection of Norwegian Speed Limit Signs", In proc. of 16th European Simulation Multiconference (ESM-2002), Darmstadt, Germany, pp.337-340 (June 2002)

20) S. Vitabile, G. Pollaccia, G. Pilato, E. Sorbello: "Road Signs Recognition Using a Dynamic pixel aggregation Technique in the HSV Color Space", in Proc. of 11th Int. Conference on Image Analysis \& Process., pp.572-577, Palermo, Italy (Sep. 2001)

21) Thi Thi Zin, C. Liao, T. Kaneko, Y. Yanagihara, H. Hama: "Recognition of Road Environment by Using Color Information from Moving Video Images", in Proc. of the 7th Image Media Process. Symposium (IMPS2002), pp.109-110 (Nov. 2002)
22) Marvin Minsky and Seymour Papert: "Perceptrons", MIT Press (1969)

23) Masamichi Shimura: "Pattern Recognition and Learning Machines", Syokodo Press (1970)

24) Thi Thi Zin, C. Liao, T. Kaneko, H. Hama, Pyke Tin: "Robust Road Sign Recognition by Using Multi-feature Template Matching", in Proc. of 2nd Int. Conference on Comput. Applications, Yangon, Myanmar, pp.474-481 (Jan. 2004)

25) Thi Thi Zin and H.Hama: "Robust Road Sign Recognition Using Standard Deviation", 7th Int. IEEE Conference on Intelligent Transportation Syst. (ITSC2004), Washington,D.C., pp.429-434 (Oct. 2004)

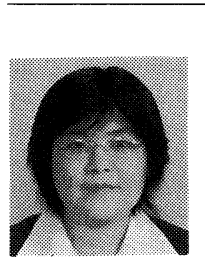

Thi Thi Zin received the B.Sc. and M.I.Sc. degrees from Yangon University and University of Computer Studies, Yangon, Myanmar in 1995 and 1999, respectively. She received the M.E. degree from Osaka City University in 2004. She is a doctoral candidate in Osaka City University, Japan. Her research interests are in ITS, Moving Image Processing, Mathematical Morphology and so on. She is a student member of IEICE.

Hiromitsu Hama received the Dr. Eng. degrees in Electrical Engineering from Osaka University, Japan. He is currently a professor of Graduate School of Engineering at Osaka City University. His research interests include $3 \mathrm{D}$ world reconstruction, computer vision, picture processing/understanding and so on. He is a member of IEEE, SPIE, IEICE, IIITE, and IPSJ.

Table 3 Preprocessing stages for segmentation.

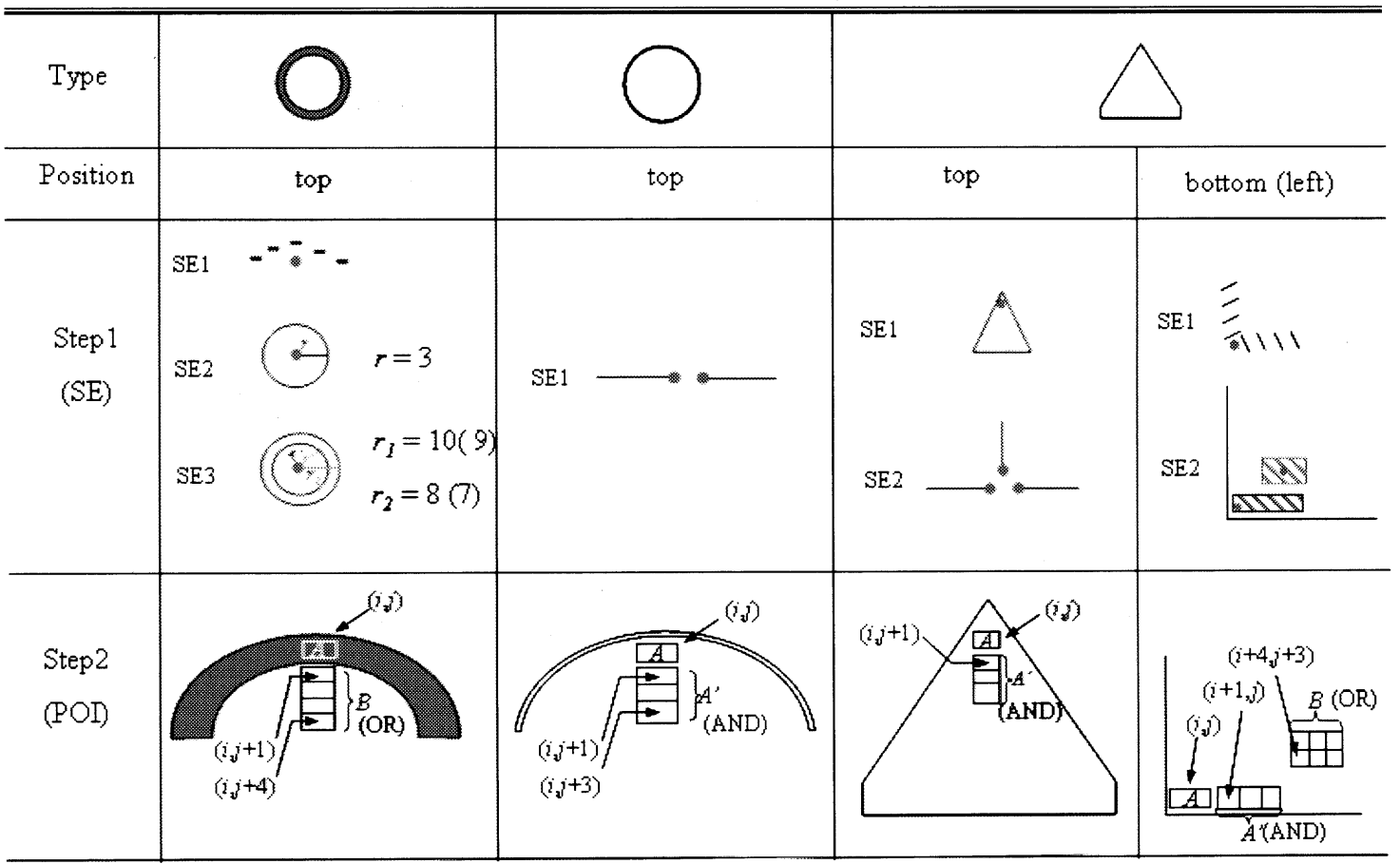

$\mathrm{POI}$ - pixel of interest

$A \quad-\mathrm{POI}\left(i_{2} j\right)$

$A$ ' - the same color region with $A$ (to satisfy ' $A N D$ ' Boolean operation)

$B$ - the different color region from $A$ (to satisfy 'OR' Boolean operation) 


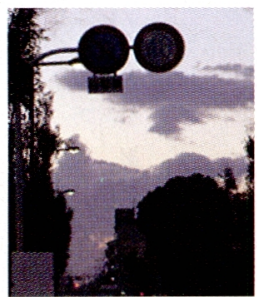

(a)

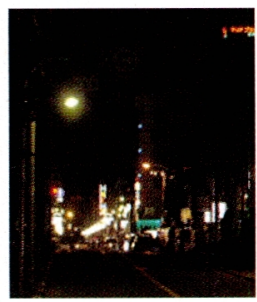

(d)

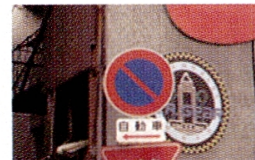

(g)

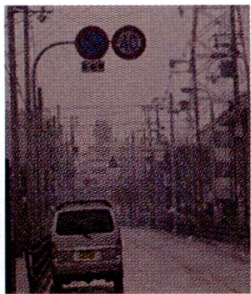

(b)

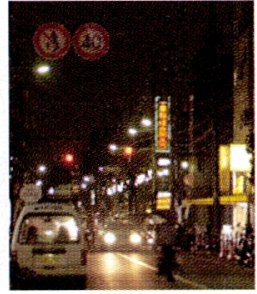

(e)

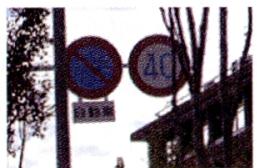

(h)

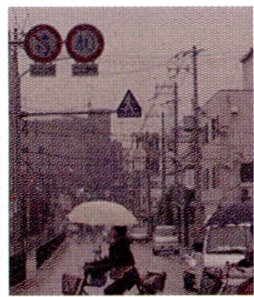

(c)

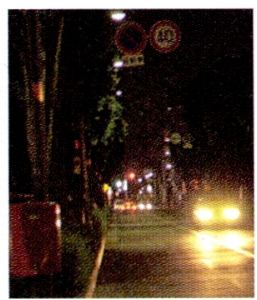

(f)

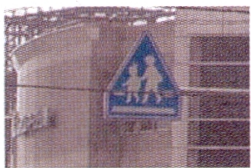

(i)
Fig.1 Examples of bad illumination conditions and difficulties: (a) dusk-twilight, (b) fog, (c) light rain, (d), (é), (f) nighttimes, (g) similar color and shape in background, (h) occlusion, (i) titled.

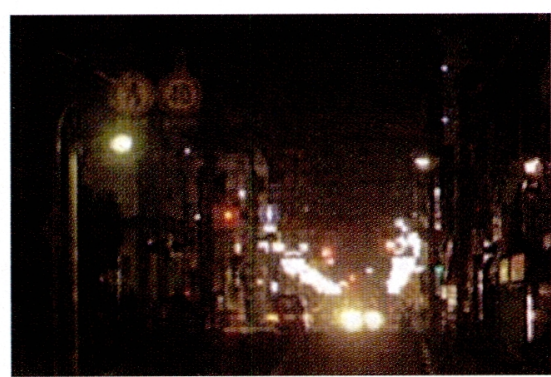

(a)

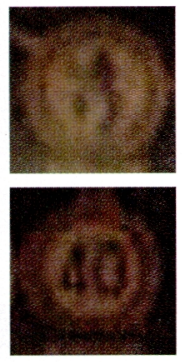

(b)
Fig.2 An examples of bad illumination condition and low resolution: (a) extremely blurred night scene with of a blustering street,

(b) manually segmented road signs from (a).

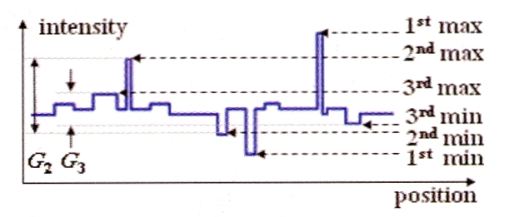

(a)

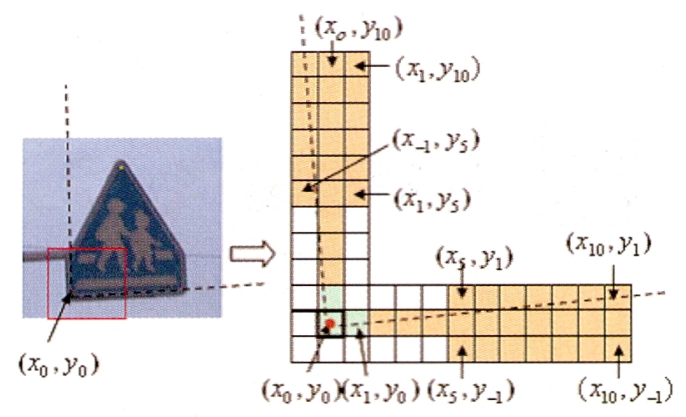

(b)

Fig.7 Examples of CMG (Type2): (a) 1D case, (b) 2D case

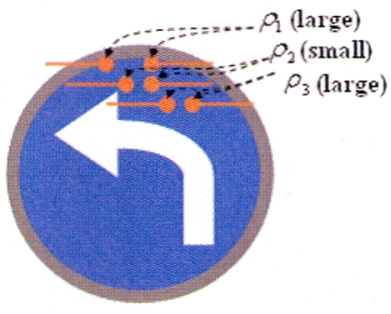

Fig.6 Example of CMG (Type1) using line shape SEs.

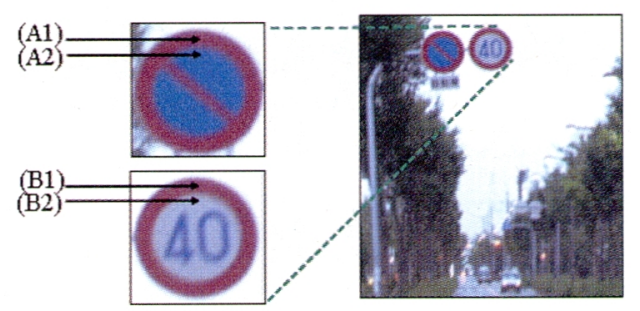

(a)

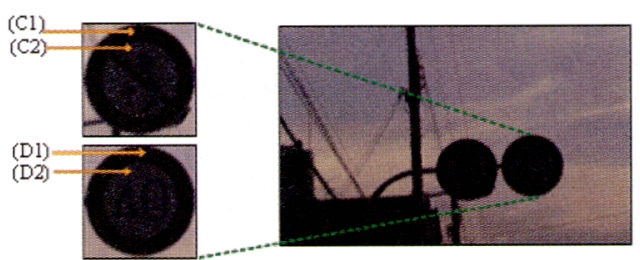

(b)

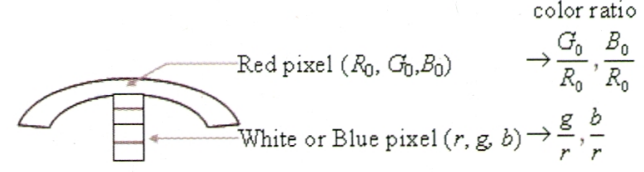

(c)

Fig.8 Examples of color composition in a road sign: (a) strongly blue color influenced images, (b) very low pixel value images, (c) relative color.

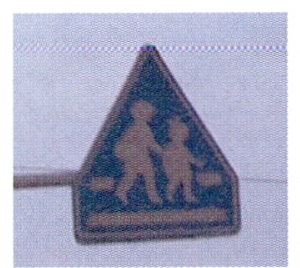

(a)

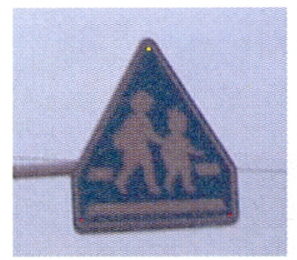

(c)

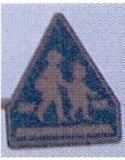

(e-i)

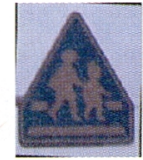

(e-ii)

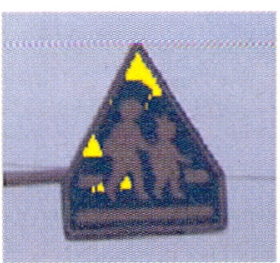

(b)

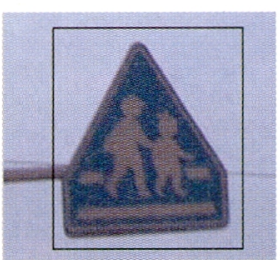

(d)
Fig.10 Examples of segmentation procedure: (a) input image, (b) nominated feature region detection using morphological operations, (c) feature point extraction using relative color from (b), (d) estimation of the exterior boundary, (e-i) first segmentation, (e-ii) rotation, (e-iii) second segmentation, (f) normalization of size. 


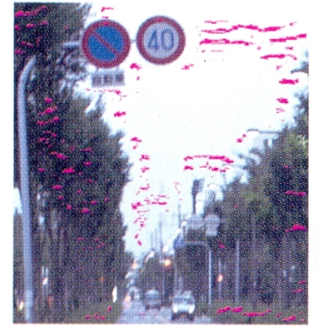

$(\mathrm{a}-\mathrm{i})$

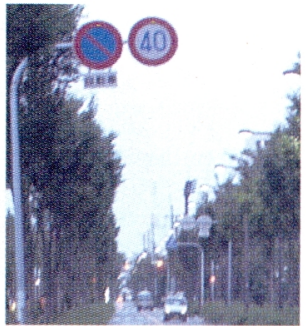

(a-ii)

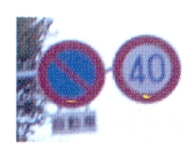

(b-i)

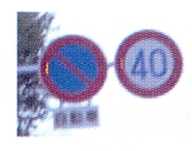

(b-ii)

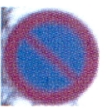

$(\mathrm{c}-\mathrm{i})$

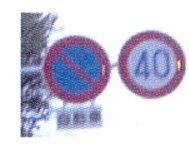

(b-iii)

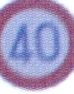

(c-ii)

Fig.11 Segmentation of road signs at evening: (a) top feature point detection: (a-i) using only morphological operations, (aii) using relative color conditions altogether; (b-i), (b-ii) and (biii) detected bottom, left and right feature points, respectively in zoomed images, (c-i) and (c-ii) segmented road signs.

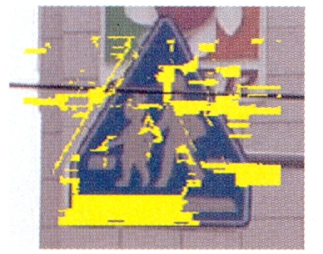

(a)

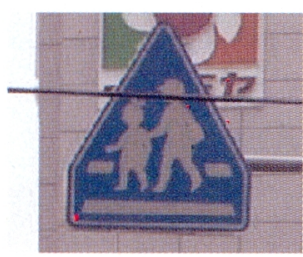

(c)

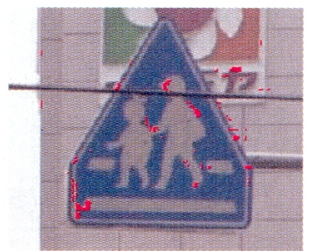

(b)

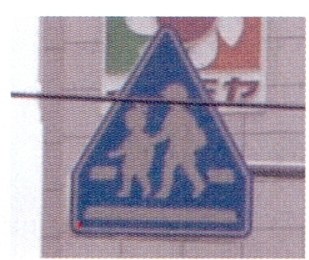

(d)

Fig.12 Pentagonal left corner detection: (a) using SE1, (b) using SE2, (c) 'AND' operation on (a) and (b), (d) using relative color on (c).

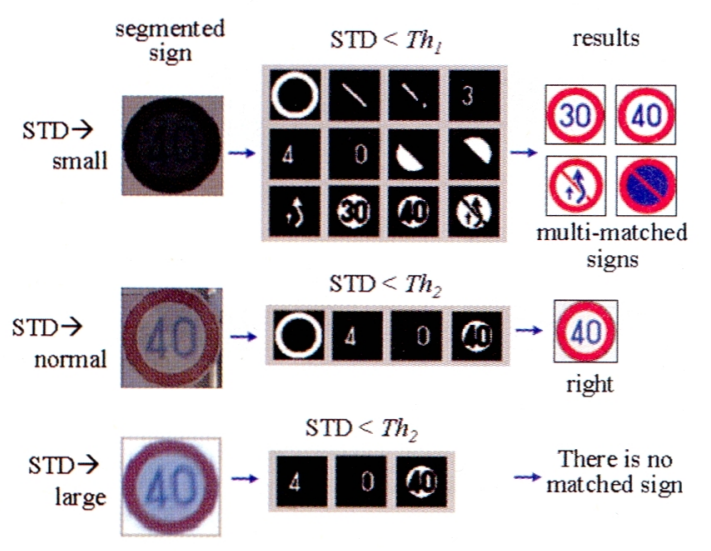

Fig.15 Template matching using only the standard deviation $S_{1}$.

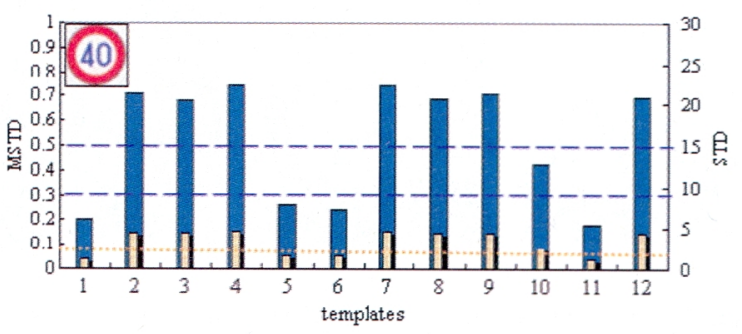

(a)

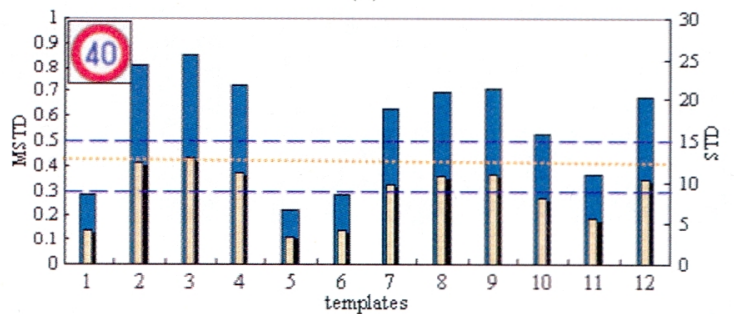

(b)

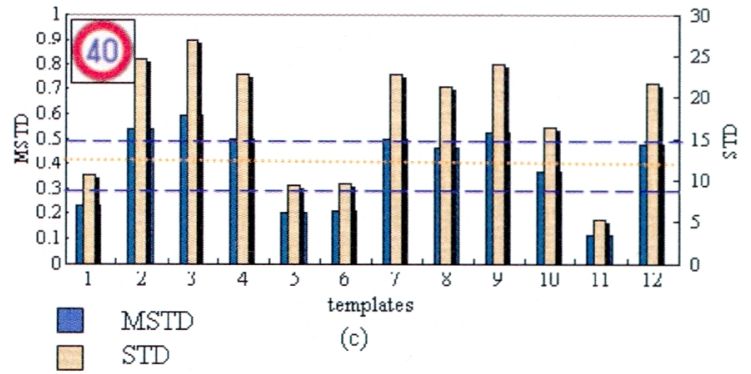

Fig.16 Template matching using MSTD: (a) small STD, (b) middle STD, (c) large STD.

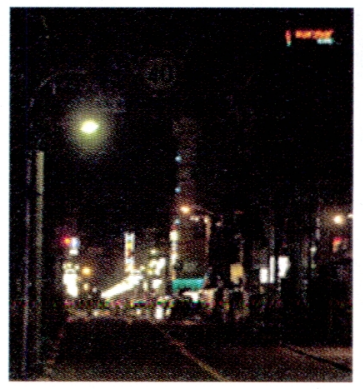

(a)

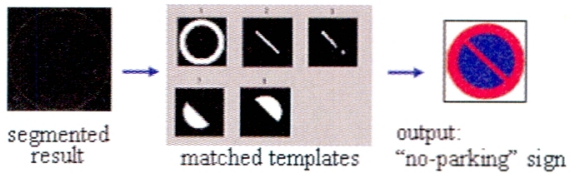

(c-i)

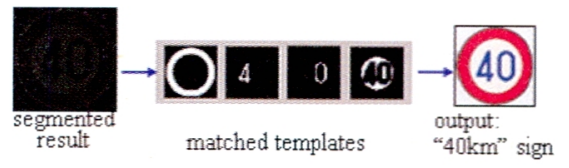

(c-ii)

Fig.17 Road sign segmentation and recognition in the nighttime: (a) input images, (b-i) and (b-ii) segmented road signs, (c-i) and (c-ii) results of template matching with MSTD of (b-i) and (b-ii), respectively. 